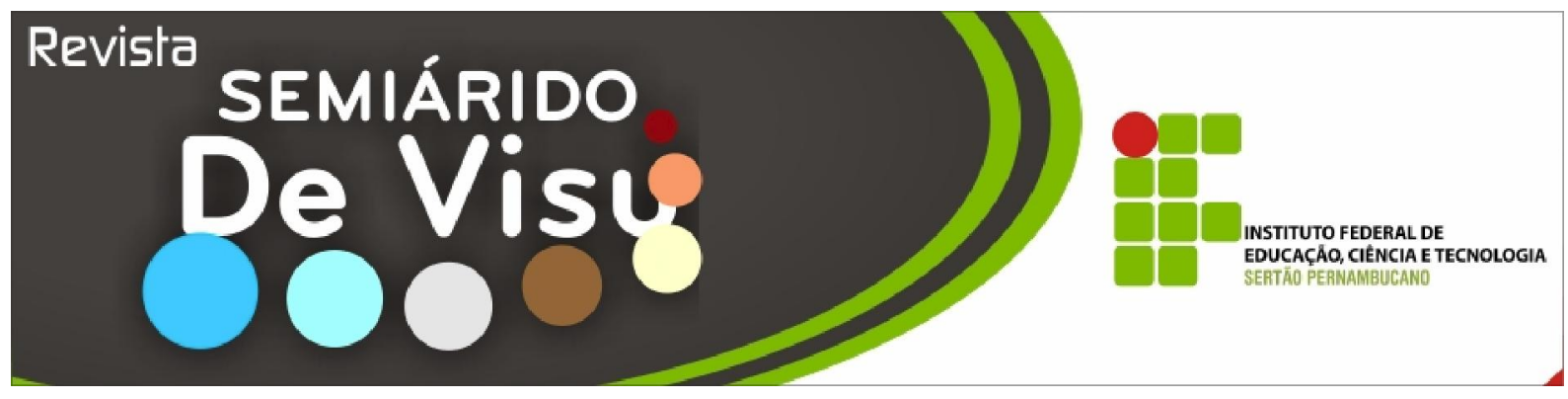

\title{
Caracterização dos térmitas na estação ecológica Raso da Catarina, Jeremoabo, Bahia
}

\author{
Carlos Alberto Batista Santos ${ }^{1}$, Rafaela Lima Gomes ${ }^{2}$ \\ ${ }^{1}$ Universidade do Estado da Bahia, Departamento de Tecnologia e Ciências Sociais, Campus III, (74) 3611 6716, Fax \\ (74) 3611 7363,E-mail: cacobatista@yahoo.com.br, rafa_biologia2010@hotmail.com
}

RESUMO: Os Isopteras, conhecidos popularmente como cupins ou térmitas, são insetos sociais que vivem em colônias constituídas por centenas, milhares e até milhões de indivíduos. Esses insetos são caracterizados como sociais, porque há divisão de tarefas entre grupos especializados, denominados castas: um par real (rei e rainha) que são os reprodutores, os operários e os soldados (estéreis). Na Caatinga a fauna de cupins ainda é pouco conhecida, em virtude de pouquíssimos estudos realizados na região Nordeste. O objetivo deste trabalho foi identificar as espécies de térmitas encontrados na Estação Ecológica Raso da Catarina no município de Jeremoabo, Estado da Bahia. A estrutura da comunidade de cupins foi avaliada em 4 pontos: Mata da Pororoca, estação 1, estação 2 e estação 3. Um protocolo padronizado com trilhas foi estabelecido em todas as áreas. 9 espécies foram encontradas, pertencentes a duas famílias distintas, Termitidae e Rhinotermitidae.

Palavras-chave: Isópteros, Caatinga, Biodiversidade

\section{Characterization of the termites in the Raso da Catarina Ecological Station, Jeremoabo, Bahia, Brazil}

\begin{abstract}
The Isopteras, popularly known as termites or termites are social insects that live in colonies consisting of hundreds, thousands and even millions of individuals. These insects are characterized as social, because there is division of labor between specialized groups, called castes: a real pair (king and queen) which are the breeding, the workers and soldiers (sterile). In the Caatinga termite fauna is poorly known due to few studies in the Northeast. The objective of this study was to identify the species of termites found in ESEC, and characterize the termite and degraded by the presence of termites. The community structure of termites was assessed at 4 points: In the woods of Pororoca, station 1, station 2 and station 3 in the ESEC (Raso da Catarina Ecological Station), located in the municipality of Jeremy, State of Bahia. A standardized protocol was established with trails in all areas. 9 species were found, belonging to two distinct families, Termitidae and Rhinotermitidae.
\end{abstract}

Keywords: Isopterans, Caatinga, Biodiversity 
Carlos Alberto Batista Santos \& Rafaela Lima Gomes

Introdução semiárido, $\begin{aligned} & \text { s Caatingas nordestinas ocupam } \\ & \text { uma área de aproximadamente } \\ & 800.000 \mathrm{~km} 2 \text { possui clima }\end{aligned}$ evapotranspiração potencial $(1500-200$ $\mathrm{mm} / \mathrm{ano}$ ) e uma baixa precipitação (300 1000/ano), concentrada em três a cinco meses (Sampaio, 1995).

O Raso da Catarina, uma das ecorregiões do bioma Caatinga, caracteriza-se por estar localizada numa bacia de solos arenosos, profundos e poucos férteis, de relevo plano, mas com canyons na parte oeste formados por afloramento de arenito. Na parte sul, Estado da Bahia, predominam os solos de areia profundos, excessivamente drenados, ácidos e de fertilidade muito baixa, encontramse também latossolos profundos, bem drenados, ácidos e de fertilidade baixa. Na parte norte, Estado de Pernambuco predomina as areias. O clima é bastante quente e seco e sua vegetação de caatinga de areia é predominantemente arbustiva, muito densa e menos espinhosa que a caatinga de solos cristalinos (Velloso et al, 2002).

Os efeitos da degradação da Caatinga sobre a estrutura das comunidades biológicas são praticamente desconhecidos, evidenciando a necessidade de estudos em áreas com diferentes níveis de impacto antrópico (Alves, 2009).

A caracterização e monitoramento da fauna silvestre podem fornecer dados consistentes sobre esses efeitos, e alguns grupos de invertebrados respondem bem às características necessárias para a realização de um monitoramento adequado.

Os insetos são considerados bons indicadores dos níveis de impacto ambiental, devido a sua grande diversidade de espécies e habitat, além da sua importância nos processos biológicos dos ecossistemas naturais (Wink et al., 2005). Os insetos sociais, em particular, estão entre os organismos mais importantes da biosfera, sendo ecologicamente dominantes, principalmente nas Regiões Tropicais (Santos \& Resende, 1996).
Os térmitas, em especial, possuem várias características que ajudam significativamente no controle de ambientes degradados, uma vez que, são insetos decompositores de material orgânico, possui grande diversidade biológica, amplitude de distribuição geográfica e são sensíveis a perturbações antrópicas, respondendo a elas em curto espaço de tempo.

Atualmente são conhecidas cerca de 2.900 espécies de térmitas sendo distribuídos em sete famílias, dessas apenas quatro famílias ocorrem no Brasil: Kalotermitidae, Rhinotermitidae, Serritermitidae e Termitidae. A abundância de suas colônias está relacionada à qualidade do micro-habitat, disponibilidade dos recursos alimentares, umidade e tipo de solo (Constantino \& Aciole, 2006).

Esses animais atuam em diversos níveis tróficos nos ecossistemas, como decompositores, auxiliam na ciclagem dos nutrientes e, através das atividades de construção, alteram as propriedades físicas e químicas do solo, podendo modificar profundamente as paisagens (Rocha \& Cancello, 2007).

A Ecologia deste grupo no Brasil ainda é pouco conhecida, em especial na região Nordeste, onde os estudos sobre esse grupo só foram efetivamente iniciados em meados da década de 1990 (Bandeira \& Vasconcellos, 1999).

O objetivo desse trabalho foi identificar a diversidade da termitofauna da Estação Ecológica Raso da Catarina em áreas similares para preservação.

\section{Material e métodos}

A ESEC Raso da Catarina está situada à margem esquerda do Rio Vaza Barris e à margem direita do Rio São Francisco a oeste da cidade de Paulo Afonso, nordeste baiano, e abrange uma área de $1.052,82 \mathrm{~km}^{2}$ (IBAMA 2005), estendendo-se sob as coordenadas 09³3', 09 $54^{\circ}$ 'S e $38^{\circ} 29^{\prime}, 38^{\circ} 44^{\prime} \mathrm{W}$ (Figura 1).

Limita-se ao norte com a aldeia Pankararé, ao oeste com Rodelas e Canudos, ao sul com Jeremoabo e ao oeste com os 
Carlos Alberto Batista Santos \& Rafaela Lima Gomes

municípios de Paulo Afonso e Jeremoabo (IBGE, 1985), todos localizados na Bahia.

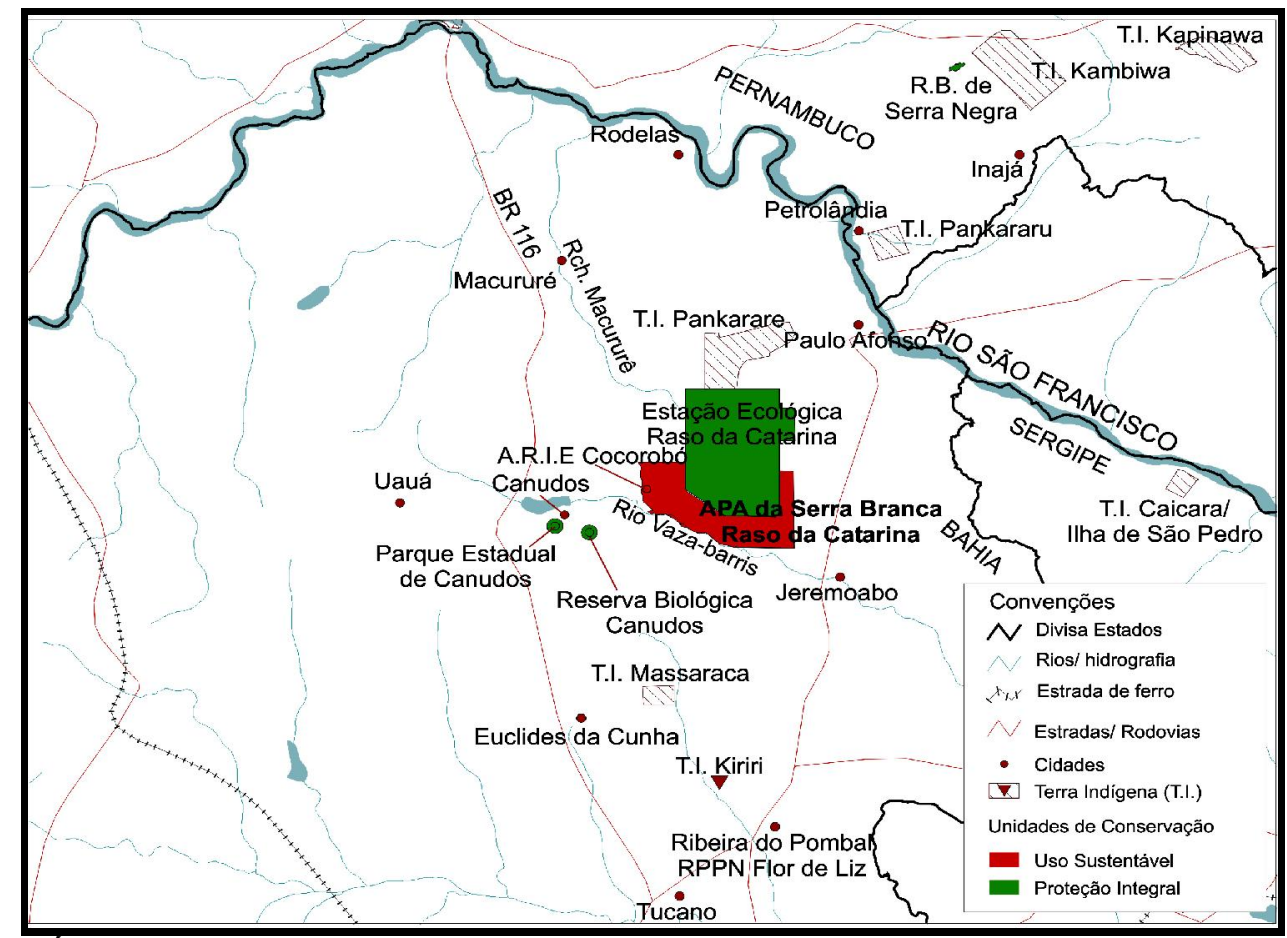

Figura 1. Área de relevante interesse ecológico, mostrando em verde a Estação Ecológica Raso da Catarina e em vermelho a APA Serra Branca/Raso da Catarina (Fonte: IBAMA, 2005).

Os cupins foram amostrados em quatro áreas selecionadas de acordo com a fitofisionomia, para amostrar faunas distintas, ao longo de quatro visitas a campo, entre julho e novembro de 2010, utilizando-se trilhas préexistentes no meio da mata, e a busca ativa em termiteiros epígios, arborícolas, troncos e galhos caídos, solo até $15 \mathrm{~cm}$ de profundidade, no folhiço, sob cascas de árvores e raízes mortas.

As áreas de coleta foram denominadas de acordo com a nomenclatura utilizada pela população local. Área 1: Mata da Pororoca; área 2: estação 1; área 3: estação 2 , e área 4: estação 3 .

A amostragem foi realizada por coleta manual com esforço amostral de 3 horas/pessoa em cada área. Esse método fornece dados qualitativos, mas é o mais eficiente e rápido para estimar a riqueza local, objetivo deste trabalho.

Os cupins foram coletados com o auxílio de pinças, fixados em álcool a 70\%, e conduzidos para identifícação taxonômica até o nível de espécie, com ajuda de bibliografia especializada, e por especialista em isópteros no laboratório da Universidade Federal do Rio Grande do Norte (UFRN), CB, Departamento de Botânica, Ecologia e Zoologia.

\section{Resultados e discussão}

Foram coletados no total, 500 indivíduos. Foram identificados nas quatro áreas de coleta: Pororoca, a estação 1 , a estação 2, e a estação 3, na ESEC Raso da Catarina um total de 9 espécies de cupins, pertencentes a duas famílias Termitidae e Rhinotermitidae, similarmente aos estudos realizado por Santos et al. (2006) no Campus da Universidade Federal de Sergipe, onde eles obtiveram também como resultado essas duas famílias.

Dos térmitas identificados, 97,87\% pertencem a Família Termitidae, corroborando com Constantino (1999), que descreve esta família como a mais abundante no mundo, compreendendo cerca de $85 \%$ das espécies 
Carlos Alberto Batista Santos \& Rafaela Lima Gomes

registradas no Brasil. A família Termitidae foi a mais abundante tendo o número mais elevado de indivíduos.

Os cupins dessa família constroem diferentes tipos de ninhos e possuem hábitos alimentares bem variados, incluindo madeira, húmus, folhas e liquens. Estudos realizado em áreas de restinga por Vasconcellos et al. (2005), também apontam a família Termitidae como a mais abundante.

Da família Termitidae foram identificados os gêneros, Nasutitermes,
Constrictotermes,

Anoplotermes, Microceroterme, Amitermes. Desses os gêneros mais representativos foram o Nasutitermes $e$ Constrictotermes, (Figura 2) confirmando os estudos feitos por Bezerra et al (2009) em área de caatinga, onde eles mostram que cupins desses gêneros são bons indicadores ecológicos por serem sensíveis à degradação do hábitat e por viverem em locais sésseis, não mudando de um hábitat para outro.

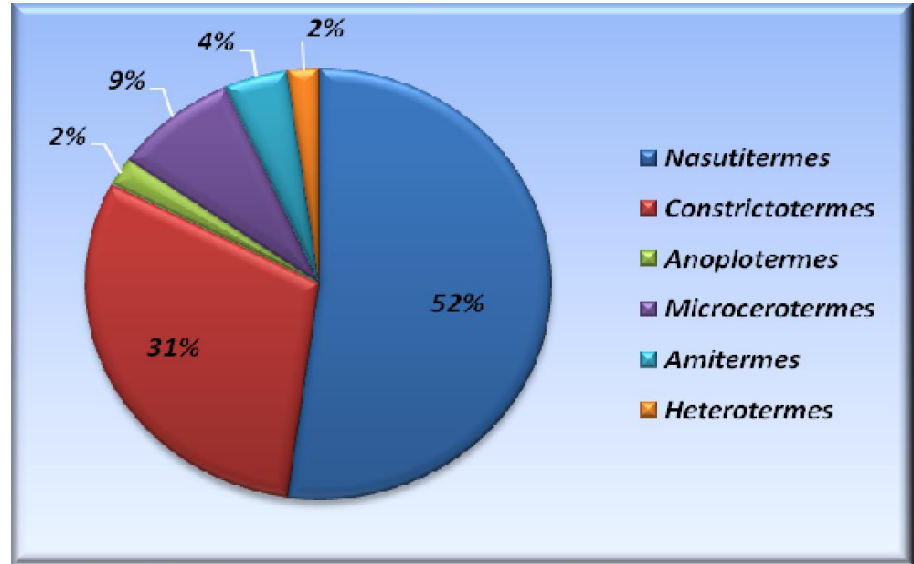

Figura 2 - Gêneros de Cupins identificados para a ESEC Raso da Catarina

Destacamos o gênero Nasutitermes, (Figura 3) com maior número de espécies encontradas, (Nasutitermes macrocephalus, Nasutitermes sp, Nasutitermes kemneri), reforçando os estudos feito por Couto et al.
(2010) em análise da termitofauna nos canaviais da Zona da mata norte e sul do estado de Pernambuco, o que demonstra a sua fácil adaptação a ambiente de Caatinga. 
Carlos Alberto Batista Santos \& Rafaela Lima Gomes

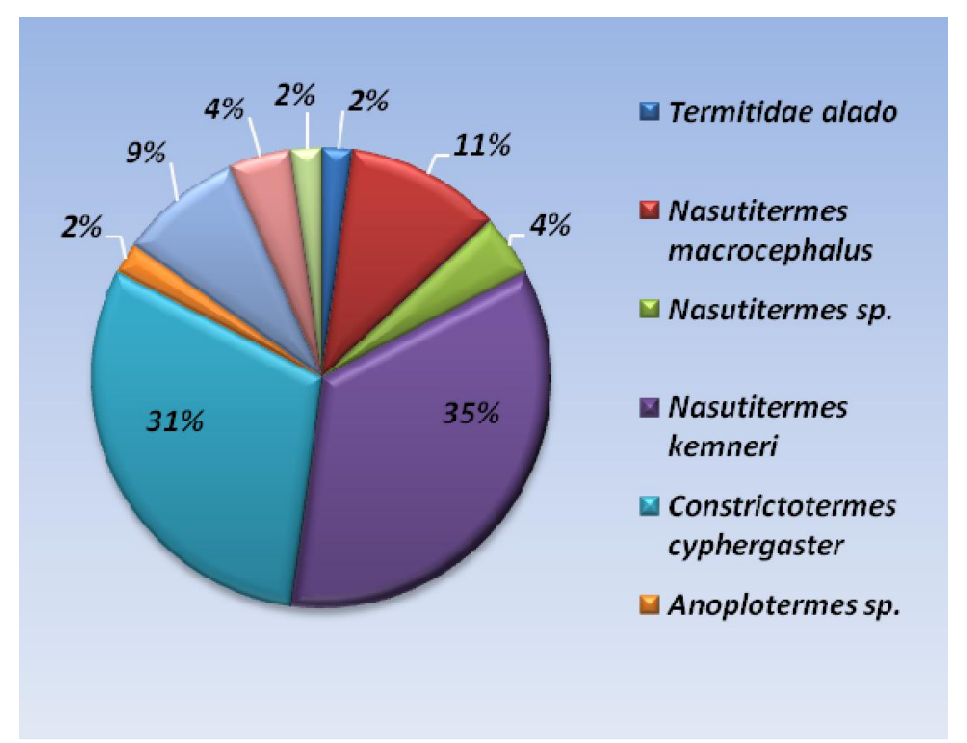

Figura 3 - Espécies Identificadas para a ESEC Raso da Catarina e sua abundância.

Das espécies catalogadas a espécie Nasutitermes kemneri foi simultânea a três das quatro áreas estudadas, excetuando-se apenas a área da Pororoca. $\mathrm{Na}$ estação 1 foram encontradas as espécies Constrictotermes cyphergaster, Nasutitermes kemneri e um indivíduo que não foi identificado da família Termitidae. Três espécies foram exclusivas da Pororoca Nasutitermes macrocephalus, Heterotermes longiceps e Amitermes amifer. $\mathrm{Na}$ estação 2 foram encontradas duas espécies Nasutitermes kemneri e Microcerotermes $s p$.

$\mathrm{Na}$ estação 3, foram coletadas quatro espécies Anoplotermes sp, Nasutitermes kemneri, Nasutitermes sp, Constrictotermes cyphergaster. Em estudo feito por (Oliveira et al, 2003) numa Transição Cerrado - Campo Rupestre, no Morro Feio, Município de Hidrolândia, Goiás, os autores registraram a variação na composição de espécies e no padrão de riqueza e abundância de cupins, as espécies Anoplotermes sp e Nasutitermes kemneri também foram apontadas. De acordo com Constantino (1992), a diversidade e composição das espécies exibem grande variabilidade, provavelmente decorrentes de diferenças metodológicas e ao esforço de coleta. A Tabela 1 trás a distribuição de espécie por área com indicação do número de indivíduos. 
Carlos Alberto Batista Santos \& Rafaela Lima Gomes

Tabela I - Distribuição de espécie por área

Número de ocorrência em cada local citado

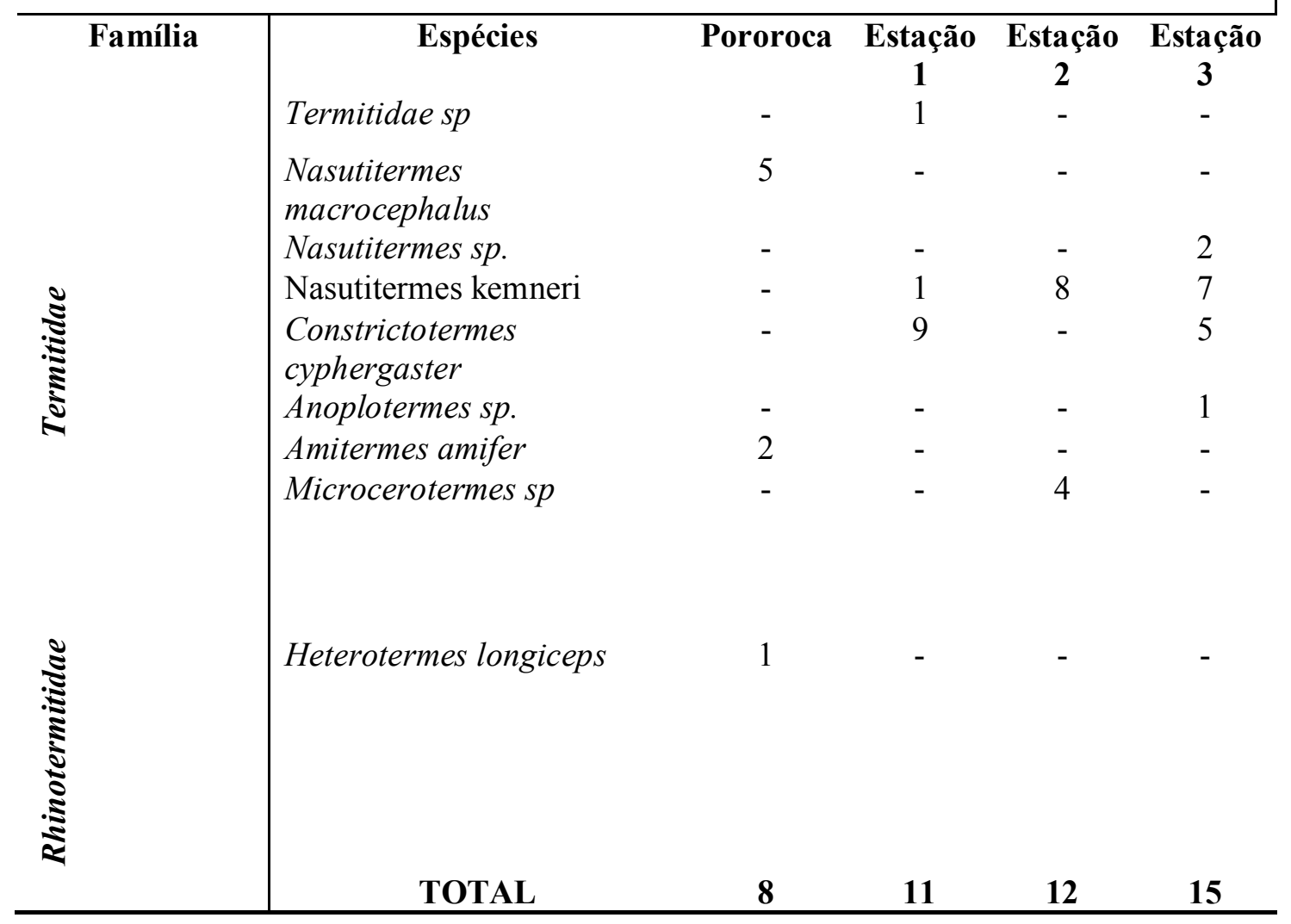

Foram encontrados $2,13 \%$ de indivíduos da família Rhinotermitidae, de um único gênero o Heterotermes, com representante na espécie Heterotermes longiceps. Cupins do gênero Heterotermes ocorrem em todas as regiões do Brasil, adaptam-se a diversos tipos de habitats e vivem, normalmente, em madeira ou em ninhos difusos no solo (Constantino, 1999). Estudos realizados por (Couto et al, 2010) em Remanescentes de Mata Atlântica inseridos em canaviais da zona da mata norte e sul do estado de Pernambuco, também apontam o gênero Heterotermes como menos abundante.

\section{Quem são os térmitas identificados na ESEC Raso da Catarina?}

\section{Família Termitidae}

É a maior família, com cerca de $70 \%$ das espécies no mundo. De acordo com Constantino et al, (2000) os térmitas da família Termitidae possuem hábitos alimentares bem variados que incluem desde madeira e húmus até folhas e liquens.

\subsection{Termitidae $s p$}

Dentre os Termitidae, alguns são comedores de madeira, de folhas, de húmus, possuindo hábitos alimentares bastante variados. Os alados apresentam semelhança geral razoavelmente grande. Também perdem as asas após a revoada, as quais podem apresentar características utilíssimas á taxonomia de alguns grupos (Filho \& Pontes, 1995).

\subsection{Nasutitermes macrocephalus}

As espécies desse grupo são pouco seletivas, pois consome madeira seca ou úmida, dura ou mole, trabalhada ou não Couto et al (2008).

\subsection{Nasutitermes $s p$}


Carlos Alberto Batista Santos \& Rafaela Lima Gomes

Estas espécies são consideradas xilófagas verdadeiras e também se alimentam de uma variedade de produtos vegetais existentes no ambiente natural, como folhas secas e troncos caídos, quando úmidos ou apodrecidos (Bandeira, 1989; Bandeira \& Macambira, 1988).

\subsection{Nasutitermes kemneri}

Pertence ao gênero Nasutitermes, alimentando-se de madeira (Vasconcellos et al, 2005). É uma espécie endêmica do cerrado e apresenta distribuição em todo o bioma, incluindo as manchas de cerrado em outros biomas (Schmidt, 2007).

\subsection{Constrictotermes cyphergaster}

Apesar dessa espécie não se alimentar da seiva e, portanto, não se caracterizar como parasita. Constrictoterme cyphergaster utiliza o solo e o húmus próximos às raízes da planta como suporte para a construção-reparo da colônia e alimentação, respectivamente, às vezes com construção de galerias subterrâneas, mas nunca conectando duas ou mais colônias (Fontes, 1980; Cunha 2000).

\subsection{Amitermes amifer}

Alimentam-se de madeira ou matéria orgânica em decomposição (Constantino, 1999). A espécie Amitermes amifer é a segunda espécie em dominância, sendo considerada praga secundária de diversas culturas (como soja, amendoim, cana-de-açúcar) (Constantino, 2009).

\subsection{Microcerotermes $s p$}

Alimentam-se de madeira e podendo ser encontrado nos mais diverso habitat (Constantino, 1999).

\subsection{Anoplotermes $s p$.}

Pertence à subfamília Apicotermitinae, uma das mais primitivas dentro do grupo dos Termitidae, é considerada de hábito intermediário, porque se alimenta de matéria orgânica do solo e madeira em estágio avançado de decomposição (Bandeira \& Macambira, 1988; Constantino, 1992).

\section{Família Rhinotermitidae}

Cupins xilófagos, que geralmente vivem em contato com o solo. São bastante abundantes e comuns, embora em poucas espécies. Várias dela são pragas importantes, em especial Coptotermes (Constantino \& Schlemmermeyer, 2000).

\subsection{Heterotermes longiceps}

Espécie xilófaga (Lee \& Wood, 1971), alimenta-se de madeira (Vasconcellos et al, 2005). Geralmente, as espécies xilófagas consomem apenas partes lignificadas de árvores vivas, incluindo o cerne.

\section{Conclusões}

A riqueza de térmitas avaliada na área de estudo mostrou-se bastante significativa, com 9 espécies encontradas, sendo $97,87 \%$ pertencente à Família Termitidae, e 2,13\% à família Rhinotermitidae, destacamos o gênero Nasutitermes, com maior número de espécies registradas para a ESEC Raso da Catarina.

A falta de conhecimento do comportamento e da biologia de cupins é um dos fatores que indicamos como sugestivo para continuar a caracterização e levantamento da termitofauna na ESEC Raso da Catarina, assim, sugerimos que novos estudos sobre termitofauna sejam implementados nesta área, incluindo aspectos da abundância das populações.

O presente trabalho contribui de forma significativa para o levantamento de cupins na região nordeste, devido à importância ecológica deste grupo de insetos e deve ser considerado como subsídio para futuros estudos sobre a termitofauna nesse ecossistema. 
Carlos Alberto Batista Santos \& Rafaela Lima Gomes

\section{Agradecimentos}

A Alexandre Vasconcelos, Professor e Pesquisador da Universidade Federal do Rio Grande do Norte, Centro de Biociências, Departamento de Botânica, Ecologia e Zoologia, pela cooperação na identificação das espécies de Térmitas.

À Equipe técnica do ICMBio, ESEC raso da Catarina, especialmente a José Tiago Almeida dos Santos e Ely Eneas F. Sousa, pelo apoio logístico durante as atividades de campo.

\section{Referências}

ALVES, G.B. Avaliação da taxa de recuperação de termiteiros arborícolas de Constrictotermes sp. (Isoptera: Termitidae) sob diferentes fatores de influência. In: IX CONGRESSO DE ECOLOGIA DO BRASIL, São Lourenço, 2009. Anais. São Lourenço, MG.

BANDEIRA, A.G. \& MACAMBIRA, M.L.J. Térmitas de Carajás, Estado do Pará, Brasil: Composição faunística, distribuição e hábito alimentar. Bol. Mus. Para. Emilio Goeldi, sér. Zool., 4(2): 175-190. 1988.

BANDEIRA, A.G. \& A. VASCONCELLOS. Estado atual do conhecimento sistemático e ecológico sobre os cupins (Insecta, Isoptera) do nordeste brasileiro. Revista Nordestina de Biologia 13:37-45. 1999.

BANDEIRA, A.G. Análise da termitofauna (Insecta: Isoptera) de uma floresta primária e de uma pastagem na Amazônia Oriental, Brasil. Bol. Mus. Para. Emílio Goeldi, sér. Zool., 5(2): 225-241. 1989.

BEZERRA, Y.B.S.; OLIVEIRA, C.R.F.; MATOS, C.H.C.; SILVA, M.L.L.S.; FERRAZ, C.S. Diversidade de cupins em áreas de caatinga em Serra Talhada-Pe. Projeto em Serra Talhada-Pe, PIBIC/CNPq bolsa de Iniciação Científica, 2009.

CONSTANTINO, R. Abundance and diversity of termites (Insecta Isoptera) in two sites of primary rain forest in Brazilian Amazonia. Biotropica, 24 (3) 420-430. 1992.

CONSTANTINO, R. Chave ilustrada para identificação dos gêneros de cupins (Insecta: Isoptera) que ocorrem no Brasil. Papéis avulsos de zoologia, v. 40, n. 25, p.387-448, 1999.

CONSTANTINO, R.; SCHLEMMERMEYER, T. Cupins (Isoptera). In: Cleber J. R. Alho. (Org.). Fauna de Manso: diagnóstico, diversidade, habitats e conservação da área sob influência do reservatório da hidrelétrica no município de Chapada dos Guimarães, Mato Grosso. Brasília: ELETRONORTE / IBAMA, 2000. p. 129-151.

CONSTANTINO, R., ACIOLI, A.N.S. Termite Diversity in Brazil, In MOREIRA, F.M.S, SIQUEIRA, J.O, BRUSSAARD, L. (org.). Soil Biodiversity in Amazonian and other Brazilian Ecosystems. Wallingford: CABI publishing. 2006. p. 117-128.

CONSTANTINO, R. Termite database. Disponível em: http://www.unb.br/ib/zoo/docente/constant/cata 1/catnew.html. Acesso em 04 junho 2010.

COUTO, A.A.V.; CASSIANO, J.K.B.; MATIAS, G.R.R.S.; SILVA, A. J. B.; ALBUQUERQUE, A. C. Estudo da Termitofauna no Bairro de Água Fria-RecifePe, Brasil. Biologia - identificação e prejuízos. Recife, Editora UFRPE, 2008. 36p.

COUTO, A.A.V. $O$ et al. Termitofauna (Insecta: Isoptera) em remanescentes de mata atlântica inseridos em canaviais da zona da mata norte e sul do Estado de Pernambuco. Recife, Editora UFRPE. 2010. 40p.

CUNHA, H.F. Estudo de colônias de Constrictotermes cyphergaster (Isoptera, Termitidae: Nasutitermitinae) no Parque Estadual da Serra de Caldas Novas, GO. Dissertação de mestrado, Univ. Federal de Goiás, Instituto de Ciências Biológicas/DBG, Goiânia. 2000. 
Carlos Alberto Batista Santos \& Rafaela Lima Gomes

FILHO, E.B. \& PONTES, L.R. Aspectos atuais da biologia e controle de cupins. Piracicaba, FEALQ, 1995. 184 p.

FONTES, E.G. Estudos ecológicos sobre o térmita arbóreo Constrictotermes cyphergaster em área de cerrado. Dissertação de Mestrado, Universidade de Brasília, Brasília. 1980.

IBAMA - Instituto Brasileiro do Meio Ambiente e dos Recursos Naturais Renováveis. 2005. Estação Ecológica Raso da Catarina. Disponível em: <// http:// www.ibama.gov.br/siucweb/mostraUC/>. Acesso em: 14 jan. 2011.

IBGE - Fundação Instituto Brasileiro de Geografia e Estatística. Cartografia: Área territorial oficial - consulta por unidade da federação. 1985. Disponível em: <//http://www.ibge.gov.br/home/geociencias/ar eaterritorial/principal.shtm $>$ Acesso em: 14 jan. 2011.

LEE, K.E. \& T.G. WOOD. 1971. Termites and Soils. London. In: LIMA, A.C. Insetos do Brasil v.1. Rio de Janeiro, Escola Nacional de Agronomia, 1938. $470 \mathrm{p}$.

OLIVEIRA, A.R.G.; FREITAS, G.A.; CHAVES, E.C.; FILHO, G. F. L, COSTA.; D.A.S.T.; CRISPIM, L.S.; BRANDÃO, D. Variação na composição de espécies e no padrão de riqueza e abundância de cupins ao longo de uma transição cerrado - campo rupestre, no Morro Feio, município de Hidrolândia, Goiás. VI CONGRESSO DE ECOLOGIA DO BRASIL, Fortaleza, 2003. Anais. Fortaleza, MG.

ROCHA, M.M. \& CANCELLO, E.M. Estudo taxonômico de Cylindrotermes Holmgren (Isoptera, Termitidae Termitinae). Pap. Avulsos Zool. v.47 n.10. 2007.

SALES. M.J.D.; MATOS. W.C.; REIS, Y. T.; RIBEIRO. G. T. Frequência e riqueza de cupins em áreas de plantio de eucalipto no litoral norte da Bahia. Pesq. agropec. bras, Brasília, v.45, n.12, p.1351-1356, dez. 2010.

SANTOS, G. I. H, RESENDE, J. J. Predação de Synterme Molestus (BURMEISTER,1839) (Isoptera-Termitidae) por Camponotus Blandus (FR. SMITH, 1858) (HyminopteraFormicidae) em Feira de Santana - BA. Sitientibus., 15:175-182. 1996.

SANTOS, B.V.C, RIBEIRO, G.T, MENDONÇA, M.C, SANTOS, H.O, DEDA, A.A.S, PASSOS, E.M. Identificação, caracterização e desenvolvimento de técnicas de controle de cupins na área do campus da UFS. In: $58^{\mathrm{a}}$ REUNIÃO ANUAL DA SBPC, Florianópolis, 2006. Anais. Florionópolis-SC. Sociedade Brasileira para o Progresso da Ciência, 1995.

SAMPAIO, E.V.S.B. Overview of de Brazilian Caatinga, In Bullock S. H.; Mooney H A \& Medina E. (eds.) Seasonally dry forest. Cambridge, Cambridge University press, Cambridge, 1995. pp. 35-58.

SCHMIDT, K. Distribuição potencial de espécie de isoptera e conservação do cerrado. Universidade de Brasília, Instituto de ciências biológicas - Programa de Pós graduação em biologia animal. 2007.

VASCONCELLOS, A.; MELO, A.C.S.; SEGUNDO, E.M.V.; BANDEIRA, A.G. Cupins de duas florestas de restinga do nordeste brasileiro. Iheringia, Sér. Zool., Porto Alegre, 95(2):127-131, 2005.

VELLOSO, A.L.; SAMPAIO, E.V.S.B.; PAREYN, F.G.C. Ecorregiões propostas para o bioma caatinga. Recife: Associação plantas do Nordeste; Instituto de Conservação Ambiental The Nature Conservancy do Brasil, 2002. 76 folhas; Il.; Fig. Mapas.

WINK, C.; GUEDES, J. V. C, FAGUNDES, C. $\mathrm{K}$, ROVEDDER, A. P. Insetos edáficos como bioindicadores ambiental. Revista de Ciências Agroveterinárias, Lages, 4(1): 60-71. 2005. 